\title{
Superconductivity from spoiling magnetism in the Kondo lattice model
}

\author{
Mohammad Zhian Asadzadeh, Michele Fabrizio, and Federico Becca \\ Democritos Simulation Center CNR-IOM Istituto Officina dei Materiali and International \\ School for Advanced Studies (SISSA), Via Bonomea 265, 34136 Trieste, Italy
}

\begin{abstract}
We find evidence that superconductivity intrudes into the paramagnetic-to-magnetic transition of the Kondo lattice model if magnetic frustration is added. Specifically, we study by the variational method the model on a square lattice in the presence of both nearest- $(t)$ and next-nearest-neighbor $\left(t^{\prime}\right)$ hopping of the conduction electrons. We find that, when $t^{\prime} / t>0$, a $d$-wave superconducting dome emerges between the magnetic and paramagnetic metal phases and close to the compensated regime, i.e., the number of conduction electrons equals the number of localized spin- $1 / 2$ moments. Superconductivity is further strengthened by a direct antiferromagnetic exchange $J_{H}$ between the localized moments, to such an extent that we observe coexistence with magnetic order.
\end{abstract}

PACS numbers: 71.27.+a, 71.30.+h, 71.10.Fd

\section{INTRODUCTION}

The emergence of superconductivity in stronglycorrelated electron systems has become quite a common phenomenon, observed by now in a wealth of different materials that include also so-called heavy-fermion compounds, where unconventional superconductivity was first reported in $\mathrm{CeCu}_{2} \mathrm{Si}_{2} \stackrel{1}{*}$ The characteristic properties of heavy fermions derive from the coexistence of itinerant electrons and localized moments residing on partly filled $f$-shells of rare earth or actinide ions. The pairing mechanism in heavy fermions has been the subject of an intense debate, also in connection with high-temperature super-

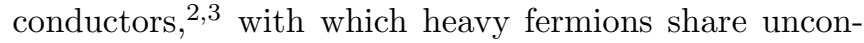
ventional pairing symmetry. For instance, recent scanning tunnelling spectroscopy on $\mathrm{CeColn}_{5}$ unveiled the presence of nodal points in the superconducting gap com-

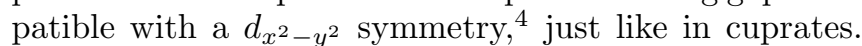
The widely accepted picture is that pairing is mediated by spin fluctuations of a nearby antiferromagnetic phase, as suggested for $\mathrm{CePd}_{2} \mathrm{Si}_{2}$ and $\mathrm{CeIn}_{3} \stackrel{5,6}{\underline{5}}$ This view is supported by the evidence that superconductivity in heavy fermions almost always appears in the vicinity of the quantum critical point that separates a paramagnetic metal phase from a magnetically-ordered one ${ }^{7}$ Even more remarkably, some compounds show a coexistence of magnetism and superconductivity, as observed in $\mathrm{CeRhSi}_{2}, \stackrel{8}{\underline{8}} \mathrm{CeRhIn}_{5}, \stackrel{9}{,}$ and $\mathrm{CeCo}\left(\operatorname{In}_{1-x} \mathrm{Cd}_{x}\right)_{5} \stackrel{10}{\underline{10}}$

From the theoretical side, the Kondo lattice model (KLM), which has been introduced by Doniach in 1977, is believed to capture the basic properties of heavy fermions. While in one spatial dimension its physical behavior is well understood, $\stackrel{12}{\rightleftharpoons}$ the more relevant twoand three-dimensional cases are much less known, especially concerning (possible) superconducting properties. Most of the analytical understanding is based upon slave bosons and large- $N$ approaches, $\stackrel{13}{=}$ which find that $d$-wave superconductivity can be indeed stabilized in two dimensions through the resonating-valence bond (RVB) mechanism, similarly to what has been suggested long ago by Anderson for high-temperature superconductors. ${ }^{14}$ As far as numerical calculations are concerned, unfortunately quantum Monte Carlo methods suffer from sign problems away from the compensated regime (where the number of itinerant electrons equals the number of localized spin-1/2 moments), while exact diagonalization and density-matrix renormalization group (DMRG) are limited to small clusters. Nonetheless, DMRG calculations suggest that the standard KLM does not support superconductivity 15 This is also the conclusion of variational Monte Carlo calculations, which show that $d$-wave pairing is indeed present in the paramagnetic sector of the KLM, but it is easily defeated by magnetism. $\frac{16}{} \mathrm{In}$ stead, recent dynamical mean-field theory (DMFT) results obtained some evidence for an unexpected $s$-wave superconductivity close to the compensated regime and relatively large Kondo coupling $\underline{\underline{17}}$

In general, the weakness of superconductivity seems the consequence of the strength of magnetism, reinforced in the model calculation by the bipartite square lattice and by the unfrustrated hopping. Since lack of magnetic frustration is rather exceptional in real materials, it is worth and legitimate to investigate how frustration modifies the phase diagram of the KLM $\stackrel{18}{=}$ Frustration in real heavy-fermion materials may take various forms. In certain cases, it can appear as a direct geometric frustration, as in the pyrochlore material $\operatorname{Pr}_{2} \mathrm{Ir}_{2} \mathrm{O}_{7} \frac{19}{\underline{P}}$ and in the Shastry-Sutherland lattice compound $\mathrm{Yb}_{2} \mathrm{Pt}_{2} \mathrm{~Pb}, \underline{20}$ in others by competing interactions of various kinds. The role of frustration in the KLM has been investigated in different works, especially focusing on magnetic properties ${ }^{21-24}$ More recently, dynamical cluster approximation (DCA) calculations on the periodic Anderson model also suggested that frustration may lead to $d$-wave superconductivity in the vicinity of an antiferromagnetic quantum critical point $\underline{25}$

There is another ingredient worth to be included to better reproduce the phase diagram of heavy fermions. Realistically, one expects that $f$-electrons are mutually coupled mostly through the conduction electrons, i.e., via the Ruderman-Kittel-Kasuya-Yosida (RKKY) exchange. However, most of the approximate methods used to study the KLM are unable to account for the RKKY interac- 
tion, unless long-range magnetic order is explicitly assumed. This inserts a bias in the calculations, since magnetic solutions can profit from the RKKY interaction, while non-magnetic ones cannot even exploit short-range magnetic correlations, e.g., to stabilize superconductivity. A way to mitigate this flaw is to add a direct $f-f$ exchange $J_{H}$ mimicking the actual RKKY interaction. The role of $J_{H}$ for reproducing the magnetic properties of heavy fermions has been highlighted several times $\underline{26}-\underline{31}$ Moreover, there are also suggestions that $J_{H}$ may be important to understand superconductivity in $\mathrm{UPd}_{2} \mathrm{Al}_{3} \underline{32}^{32}$

In this paper, we show that the underlying superconducting properties of the Kondo lattice model naturally emerge whenever magnetism is frustrated and/or local Cooper pairs are reinforced by a direct $f-f$ exchange. Here, the mechanism leading to superconductivity can be captured by the standard approach where spin fluctuations mediate pairing; however, without these extra ingredients, pairing is not strong enough to overcome magnetic long-range order. One of the main signatures of this mechanism is the $d_{x^{2}-y^{2}}$ symmetry of the order parameter, which arises from the indirect coupling of conducting electrons through localized moments.

The paper is organized as follow: in Sec. III we introduce the KLM and describe the numerical method, in Sec. [II], we present the results, and finally, in Sec. [V] we draw our conclusions.

\section{MODEL AND METHOD}

We explore the phase diagram of an extended KLM on a square lattice described by the Hamiltonian:

$$
\begin{aligned}
\mathcal{H}= & -t \sum_{\langle i, j\rangle, \sigma}\left(c_{i, \sigma}^{\dagger} c_{j, \sigma}+\text { h.c. }\right)-t^{\prime} \sum_{\langle\langle i, j\rangle\rangle, \sigma}\left(c_{i, \sigma}^{\dagger} c_{j, \sigma}+\text { h.c. }\right) \\
& +J \sum_{i} \mathbf{S}_{i} \cdot \mathbf{s}_{i}+J_{H} \sum_{\langle i, j\rangle} \mathbf{S}_{i} \cdot \mathbf{S}_{j},
\end{aligned}
$$

where $\langle i, j\rangle$ and $\langle\langle i, j\rangle\rangle$ imply that $i$ and $j$ are nearest neighbors and next-nearest neighbors, respectively; $\mathbf{S}_{i}$ is the spin 1/2-operator of the local moment at site $i$, and $\mathbf{s}_{i}$ that of the conduction electrons. Hereafter, we shall refer to the frustrated Kondo lattice model (FKLM) when $t^{\prime} \neq 0$ but $J_{H}=0$, and to the Kondo-Heisenberg lattice model (KHLM) in the opposite case of $t^{\prime}=0$ but $J_{H} \neq 0$. We take $t=1$ as the energy unit, and study the phase diagram in the the uncompensated regime, where the density of $c$-electrons $n_{c}<1$, by varying the frustrating hopping $t^{\prime}$, the Kondo coupling $J$, and the superexchange $J_{H}$.

We study the ground state of Eq. (1) by variational Monte Carlo technique. The variational wave function is defined by:

$$
\left|\Psi_{v}\right\rangle=\mathcal{P}_{f}\left|\Phi_{\mathrm{MF}}\right\rangle,
$$

where $\mathcal{P}_{f}$ is the Gutzwiller projector that enforces single occupancy of $f$ electrons on each site, while $\left|\Phi_{\mathrm{MF}}\right\rangle$ is an uncorrelated wave function defined as the ground state of a non-interacting variational Hamiltonian, $\mathcal{H}_{\mathrm{MF}}$, that in general contains as variational parameters $c-c, c-f$ and $f-f$ hybridization terms, an energy shift of the $f$ orbitals, staggered magnetic fields acting on $c$ - and $f$ electrons, as well as BCS coupling terms $\underline{\underline{16}}$ Depending on $\mathcal{H}_{\mathrm{MF}}$ we can describe different uncorrelated states:

1) A paramagnetic normal metal, which we denote by PM:

$$
\mathcal{H}_{\mathrm{PM}}=\sum_{k, \sigma}\left[\begin{array}{ll}
c_{k, \sigma}^{\dagger} & f_{k, \sigma}^{\dagger}
\end{array}\right]\left[\begin{array}{cc}
\chi_{k}^{c c} & V \\
V & \chi_{k}^{f f}
\end{array}\right]\left[\begin{array}{c}
c_{k, \sigma} \\
f_{k, \sigma}
\end{array}\right] .
$$

2) A singlet superconductor with inversion symmetry, denoted by $\mathrm{PM}+\mathrm{BCS}$ :

$$
\begin{array}{r}
\mathcal{H}_{\mathrm{PM}+\mathrm{BCS}}=\sum_{k}\left[\begin{array}{lll}
c_{k, \uparrow}^{\dagger} & c_{-k, \downarrow} & f_{k, \uparrow}^{\dagger} \\
f_{-k, \downarrow}
\end{array}\right] \times \\
{\left[\begin{array}{cccc}
\chi_{k}^{c c} & \Delta_{k}^{c c} & V & \Delta_{k}^{c f} \\
\Delta_{k}^{c c} & -\chi_{k}^{c c} & \Delta_{k}^{c f} & -V \\
V & \Delta_{k}^{c f} & \chi_{k}^{f f} & \Delta_{k}^{f f} \\
\Delta_{k}^{c f} & -V & \Delta_{k}^{f f} & -\chi_{k}^{f f}
\end{array}\right]\left[\begin{array}{c}
c_{k, \uparrow} \\
c_{-k, \downarrow}^{\dagger} \\
f_{k, \uparrow} \\
f_{-k, \downarrow}^{\dagger}
\end{array}\right] .}
\end{array}
$$

3) An antiferromagnetic metal:

$$
\begin{aligned}
\mathcal{H}_{\mathrm{AF}}= & \sum_{k \in M B Z, \sigma}\left[\begin{array}{llll}
c_{k, \sigma}^{\dagger} & c_{k+Q, \sigma}^{\dagger} & f_{k, \sigma}^{\dagger} & f_{k+Q, \sigma}^{\dagger}
\end{array}\right] \times \\
& {\left[\begin{array}{cccc}
\chi_{k}^{c c} & m_{f} \sigma & V & 0 \\
m_{f} \sigma & -\chi_{k}^{c c} & 0 & V \\
V & 0 & \chi_{k}^{f f} & m_{c} \sigma \\
0 & V & m_{c} \sigma & -\chi_{k}^{f f}
\end{array}\right]\left[\begin{array}{c}
c_{k, \sigma} \\
c_{k+Q, \sigma} \\
f_{k, \sigma} \\
f_{k+Q, \sigma}
\end{array}\right], }
\end{aligned}
$$

where the sum over $k$ is restricted to the reduced Brillouin zone and $Q=(\pi, \pi)$. In the latter case, two possible states can be variationally obtained that differ by the topology of their Fermi surface, 33 either electron- or holelike, which we refer to as $\mathrm{AFe}$ and $\mathrm{AFh}$, respectively.

In all the previous cases, $\chi_{k}^{c c}=-2\left(\cos k_{x}+\cos k_{y}\right)-$ $4 \tilde{t}^{\prime} \cos k_{x} \cos k_{y}$ and $\chi_{k}^{f f}=-2 \tilde{t}_{f}\left(\cos k_{x}+\cos k_{y}\right)-\mu_{f} ; \tilde{t}^{\prime}$, $\tilde{t}_{f}$, and $\mu_{f}$ being variational parameters, as well as hybridization $V$ and magnetizations for $c$ and $f$ electrons $\left(m_{c}\right.$ and $\left.m_{f}\right)$. The best variational energies are obtained allowing a superconducting pairing with $d_{x^{2}-y^{2}}$ symmetry, compared to on-site or extended $s$-wave symmetry. The most relevant ones are $\Delta_{k}^{c c}=2 \Delta_{c}\left(\cos k_{x}-\cos k_{y}\right)$ and $\Delta_{k}^{f f}=2 \Delta_{f}\left(\cos k_{x}-\cos k_{y}\right)\left(\Delta_{c}\right.$ and $\Delta_{f}$ being additional variational parameters).

4) Whenever antiferromagnetism coexist with superconductivity, we label the states as $\mathrm{AFh}+\mathrm{BCS}$ or $\mathrm{AFe}+\mathrm{BCS}$ (we do not write the explicit form of the $8 \times 8$ matrix representing the uncorrelated Hamiltonian).

We would like to mention that we do not consider magnetic states with incommensurate or inhomogeneous patterns, since, in general, incommensurate spirals are fragile and do not give rise to sizable energy gains, while 

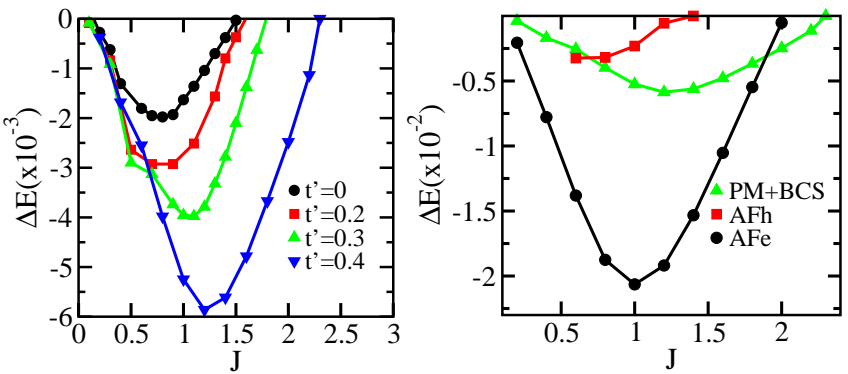

FIG. 1: (Color on-line) Left panel: energy (per site) difference between the superconducting state and the paramagnetic metal as a function of the Kondo coupling $J$ for different values of positive $t^{\prime}$. Right panel: energy (per site) difference of magnetic and superconducting phases with respect to paramagnetic metal versus $J$ for $t^{\prime}=0.4$. The cluster has $L=8$ and the density of conduction electrons is $n_{c} \simeq 0.94$.
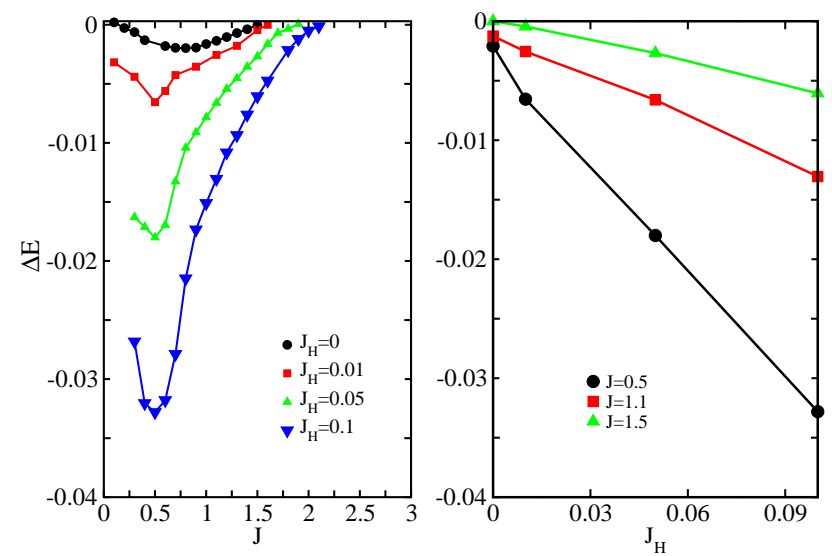

FIG. 2: (Color on-line) Left panel: superconducting condensation energy (per site) versus the Kondo exchange $J$ for different values of $J_{H}$ and $n_{c} \simeq 0.94$. Right panel: the same quantity for fixed value of $J$ versus $J_{H}$.

inhomogeneous phases are not expected in the antiferromagnetic Kondo lattice model (on the contrary, the latter ones have been found in models with ferromagnetic Kondo super-exchange) $\stackrel{34.35}{=}$ The variational parameters are obtained by minimizing the total energy by quantum Monte Carlo simulations $\stackrel{36}{ }$ Calculations have been performed on $L \times L$ square clusters with $L$ ranging from 8 to 20 .

\section{RESULTS}

We start by discussing the results for the FKLM of Eq. (11) with $J_{H}=0$. First, we focus on the paramagnetic sector, namely on PM and PM+BCS states. We report in Fig. 1 the condensation energy, computed as the energy difference between the optimized superconducting state and the best paramagnetic metal, for $n_{c} \simeq 0.94$
TABLE I: Energies per site of the paramagnetic metal and the best superconducting state for $t^{\prime}=0.4, J=2.1$ and $n_{c} \simeq 0.94$ on $L \times L$ clusters.

\begin{tabular}{ccccc}
\hline$L$ & $n_{c}$ & $E^{P M}$ & $E_{P M+B C S}$ & $\Delta E=E_{P M+B C S}-E_{P M}$ \\
\hline \hline 8 & 0.937 & $-2.18660(2)$ & $-2.18912(2)$ & $-0.00252(4)$ \\
12 & 0.930 & $-2.18274(2)$ & $-2.18429(2)$ & $-0.00155(4)$ \\
14 & 0.938 & $-2.18721(2)$ & $-2.18866(2)$ & $-0.00145(4)$ \\
16 & 0.937 & $-2.18650(2)$ & $-2.18784(2)$ & $-0.00134(4)$ \\
18 & 0.926 & $-2.18016(2)$ & $-2.18126(2)$ & $-0.00110(4)$ \\
20 & 0.940 & $-2.18782(2)$ & $-2.18910(2)$ & $-0.00128(4)$ \\
\hline \hline
\end{tabular}

and different values of $t^{\prime}$. The presence of a positive nextnearest-neighbor $t^{\prime}$ is found to considerably enhance the condensation energy, while negative values considerably suppresses it (not shown). The enlargement of the stability region of superconductivity for $t^{\prime}>0$ is also remarkable: while for $t^{\prime}=0$ the condensation energy vanishes for $J \simeq 1.5$, for $t^{\prime}=0.4$ superconductivity survives up to $J \simeq 2.3$.

Let us now consider magnetic states. We recall that, in the absence of frustration, the magnetic solution has always lower energy than the superconducting one, when the latter is stable, hence the actual phase diagram does not include superconductivity at all 16,33 This situation changes when frustration is added. In Fig. 1, we report the optimized energy of $\mathrm{PM}+\mathrm{BCS}, \mathrm{AFh}$, and $\mathrm{AFe}$ states relative to the $\mathrm{PM}$ one, for $t^{\prime}=0.4$ and $n_{c} \simeq 0.94$ (in this case, we do not find any appreciable gain by allowing both magnetism and superconductivity). The first observation is that the AFh state is strongly hindered by $t^{\prime}$, while the AFe state lowers its energy. The most important feature is that now the superconducting state takes over antiferromagnetism in a wide range of parameters.

The existence of a superconducting phase close to the paramagnetic to magnetic transition is confirmed by performing the size scaling up to $L=20$. In Table [. we report the energies of the paramagnetic metal and the best superconducting state (as well as their difference) for $t^{\prime}=0.4, J=2.1$, and $n_{c} \simeq 0.94$. Due to finite-size effects, it is not possible to fix exactly the same value of $n_{c}$ for all clusters, nevertheless, the differences in the electron concentration is very similar in all cases. Although the condensation energy is quite reduced from $L=8$ to $L=12$, it remains essentially constant from $L=12$ to $L=20$, indicating a finite value in the thermodynamic limit. In light of these results, we believe that a true superconducting phase does exist in the vicinity of the magnetic transition (especially for large values of $J$ and $t^{\prime}$ ). Finally, further away from compensated regime (i.e., for $\left.n_{c} \lesssim 0.8\right)$ the superconducting phase is defeated by the AFe state, and eventually disappears.

We now turn to the KHLM, with $t^{\prime}=0$ but $J_{H}>0$ in Eq. (11). We observe that a variational wave function could in principle account for the RKKY exchange, hence not require any $J_{H}$, through spin-spin Jastrow factors. 

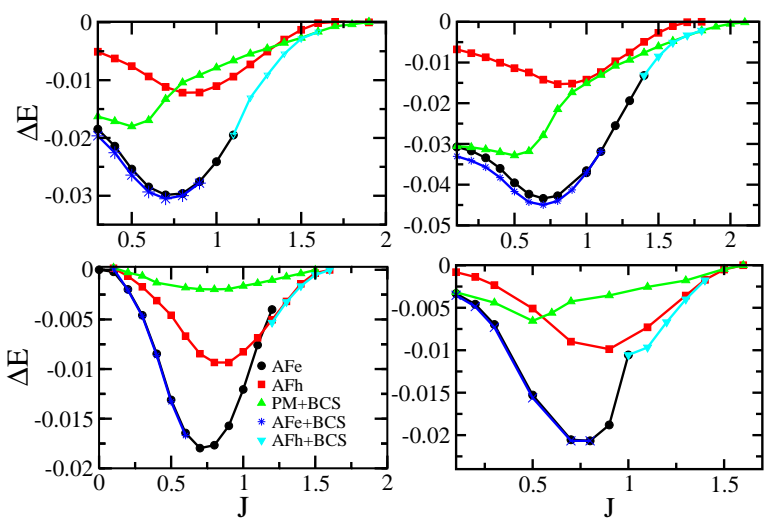

FIG. 3: (Color on-line) Energy (per site) difference of magnetic and superconducting phases with respect to paramagnetic state as a function of the Kondo exchange $J$, for different values of $J_{H}$ and $n_{c} \simeq 0.94$. The cases with $J_{H}=0$ (bottom left), $J_{H}=0.01$ (bottom right), $J_{H}=0.05$ (top left), and $J_{H}=0.1$ (top right) are reported.
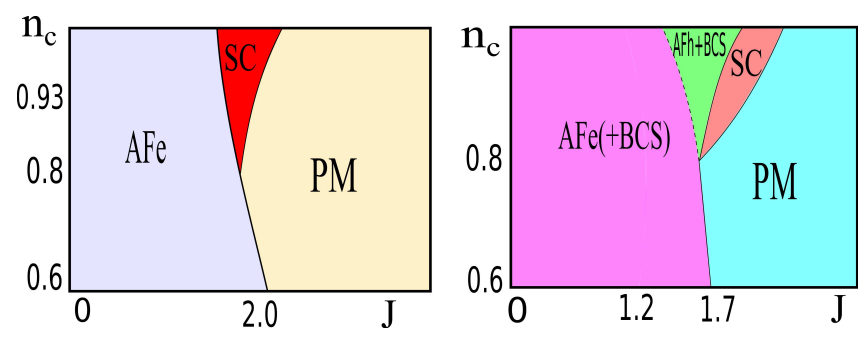

FIG. 4: (Color on-line) Schematic phase diagrams in the plane of $n_{c}$ and $J$ for frustrated Kondo lattice model (left panel, with $t^{\prime}=0.4$ ) and Kondo-Heisenberg lattice model (right panel, with $J_{H}=0.1$ ).

However, in practice this is unfeasible unless spin $S U(2)$ symmetry is explicitly broken. Therefore, even though our variational approach is more accurate than HartreeFock, we still need to include a direct $f-f$ exchange to add magnetic short-range correlations provided in reality by the RKKY interaction.

As before, we start by the paramagnetic sector. Also in this case the superconducting pairing has $d_{x^{2}-y^{2}}$ symmetry. In Fig. 2, we show the condensation energy for different values of $J_{H}$ at $n_{c} \simeq 0.94$. The case of $J_{H}=0$ has been also reported for comparison. The maximum gain remains peaked around $J=0.5$ but increases monotonically with $J_{H}$. Remarkably, even tiny values of $J_{H}$ substantially enhance the condensation energy. The inclusion of $J_{H}$ not only enlarges the condensation energy but also the stability region of superconductivity. While at $J_{H}=0$ the transition to a normal metal occurs at $J \simeq 1.5$, for $J_{H}=0.1$ the superconducting state remains stable up to $J \simeq 2.2$.

To assess whether superconductivity does exist in the phase diagram, we now examine also magnetic states. Obviously, a direct antiferromagnetic interaction $J_{H}$ en-
TABLE II: Energies per site of the paramagnetic metal and the best superconducting state for $J_{H}=0.1, J=1.8$ and $n_{c} \simeq 0.94$ on $L \times L$ clusters.

\begin{tabular}{ccccc}
\hline$L$ & $n_{c}$ & $E^{P M}$ & $E_{P M+B C S}$ & $\Delta E=E_{P M+B C S}-E_{P M}$ \\
\hline \hline 8 & 0.937 & $-1.99355(2)$ & $-1.99570(2)$ & $-0.00215(4)$ \\
12 & 0.930 & $-1.98825(2)$ & $-1.98961(2)$ & $-0.00136(4)$ \\
14 & 0.938 & $-1.99448(2)$ & $-1.99584(2)$ & $-0.00136(4)$ \\
16 & 0.937 & $-1.99353(2)$ & $-1.99469(2)$ & $-0.00116(4)$ \\
18 & 0.926 & $-1.98471(2)$ & $-1.98558(2)$ & $-0.00087(4)$ \\
20 & 0.940 & $-1.99539(2)$ & $-1.99645(2)$ & $-0.00106(4)$ \\
\hline \hline
\end{tabular}

hances the tendency towards Néel order, hence enlarges the stability region of antiferromagnetism. In Fig. 3, we show the energy of magnetic and superconducting states relative to the paramagnetic state, for different values of $J_{H}$ and $n_{c} \simeq 0.94$. The case $J_{H}=0$ has been also included for comparison. Interestingly, upon increasing $J_{H}$ the superconducting phase finally gets energetically more favorable than AFh. The stability of a pure superconducting phase close to the paramagnetic to magnetic transition is confirmed by a size scaling of the condensation energy, see Table II. Also in this case, all evidences point to a condensation energy that remains finite in the thermodynamic limit. Therefore, a superconducting region in the vicinity of the magnetic quantum critical point emerges as before, this time thanks to a finite $J_{H}$.

Furthermore, $J_{H}$ also stabilizes coexistence of pairing and magnetism, especially close to the quantum critical point. Indeed, we find a substantial energy gain when adding superconducting parameters on top of the AFh state, giving rise to a AFh+BCS phase. In other words, upon reducing the Kondo coupling, the paramagnetic metal first becomes superconducting through a secondorder transition and then acquires magnetic order, still displaying a sizable electron pairing, see Fig. 3. By further reducing the Kondo exchange, a first-order transition to a AFe state occurs. Its energy can be slightly lowered by allowing for a BCS coupling, see Fig. 3, This gain decreases with increasing $L$, possibly indicating that the coexistence of magnetism and superconductivity will disappear in the thermodynamic limit, see Table III.

For lower electron densities, namely for $n_{c} \lesssim 0.8$ the AFh state cannot be stabilized anymore, similarly to the $J_{H}=0$ case. In addition, the pure superconducting phase $\mathrm{PM}+\mathrm{BCS}$ is now defeated by the AFe state, although the latter may still allow for a coexisting superconductivity.

\section{CONCLUSIONS}

In summary, we have shown that magnetic frustration in the Kondo lattice model has the important role of suppressing magnetic order hence uncovering superconductivity, which we find intrudes between the paramagnetic 
TABLE III: Energies per site of the magnetic state (AFe) and the one with both magnetism and superconductivity for $J_{H}=0.1, J=0.5$ and $n_{c} \simeq 0.94$ on $L \times L$ clusters.

\begin{tabular}{ccccc}
\hline$L$ & $n_{c}$ & $E^{A F e}$ & $E_{A F e+B C S}$ & $\Delta E=E_{A F e+B C S}-E_{A F e}$ \\
\hline \hline 8 & 0.937 & $-1.70758(2)$ & $-1.70972(2)$ & $-0.00214(4)$ \\
12 & 0.930 & $-1.69280(2)$ & $-1.69442(2)$ & $-0.00162(4)$ \\
14 & 0.938 & $-1.69733(2)$ & $-1.69877(2)$ & $-0.00144(4)$ \\
16 & 0.937 & $-1.69240(2)$ & $-1.69353(2)$ & $-0.00113(4)$ \\
18 & 0.926 & $-1.69588(2)$ & $-1.69686(2)$ & $-0.00098(4)$ \\
20 & 0.940 & $-1.69372(2)$ & $-1.69419(2)$ & $-0.00047(4)$ \\
\hline \hline
\end{tabular}

and antiferromagnetic metal phases, see Fig. 4. Superconductivity is further stabilized by short-range magnetic correlations, which in reality are yielded by the RKKY exchange but which we had to enforce in our variational calculation through a direct antiferromagnetic exchange $J_{H}$ between the localized moments. Even for quite small $J_{H}$, a superconducting dome appears between the antiferromagnet and the paramagnetic metals, for $n_{c} \gtrsim 0.8$, see Fig. 4. We also have indications for a coexistence of magnetism and superconductivity when the Kondo exchange is small.

Therefore, both the occurrence of a superconducting dome right in the vicinity of the quantum critical point separating the magnetic metal from the paramagnetic one, the typical example being $\mathrm{CePd}_{2} \mathrm{Si}_{2}, \frac{5,6}{,}$ and the coexistence of antiferromagnetism and superconductivity, observed in $\mathrm{CeRhSi}_{2}, \frac{8}{,} \mathrm{CeRhIn}_{5}, \stackrel{9}{,}$ and, more recently, $\mathrm{CeCo}\left(\operatorname{In}_{1-x} \mathrm{Cd}_{x}\right)_{5} \stackrel{10}{\rightleftharpoons}$ are reproduced by an enriched Kondo lattice model.

This work was partially supported by PRIN 2010-11.
${ }^{1}$ F. Steglich, J. Aarts, C.D. Bredl, W. Lieke, D. Meschede, W. Franz, and H. Schafer, Phys. Rev. Lett. 43, 1892 (1979).

2 M.R. Norman, Science 332, 196 (2011).

3 D.J. Scalapino, Rev. Mod. Phys. 84, 1383 (2012).

${ }^{4}$ M.P. Allan, F. Massee, D.K. Morr, J. Van Dyke, A.W. Rost, A.P. Mackenzie, C. Petrovic, and J.C. Davis, Nat. Phys. 9, 468 (2013).

5 N.D. Mathur, F.M. Grosche, S.R. Julian, I.R. Walker, D.M. Freye, R.K.W. Haselwimmer, and G.G. Lonzarich, Nature (London) 394, 39 (1998).

${ }^{6}$ F.M. Grosche, I.R. Walker, S.R. Julian, N.D. Mathur, D.M. Freye, M.J. Steiner, and G.G. Lonzarich, J. Phys.: Condens. Matter 13, 2845 (2001).

7 Q. Si and F. Steglich, Science 329, 1161 (2010).

8 R. Movshovich, T. Graf, D. Mandrus, J.D. Thompson, J.L. Smith, and Z. Fisk, Phys. Rev. B 53, 8241 (1996).

9 M. Yashima, S. Kawasaki, H. Mukuda, Y. Kitaoka, H. Shishido, R. Settai, and Y. Onuki, Phys. Rev. B 76, 020509 (2007).

10 S. Nair, O. Stockert, U. Witte, M. Nicklas, R. Schedler, K. Kiefer, J.D. Thompson, A.D. Bianchi, Z. Fisk, S. Wirth, and F. Steglich, PNAS 107, 9537 (2010).

11 S. Doniach, Physica B \& C 91, 231 (1977).

12 H. Tsunetsugu, M. Sigrist, and K. Ueda, Rev. Mod. Phys. 69, 809 (1997).

${ }^{13} \mathrm{P}$. Coleman, in Handbook of Magnetism and Advanced Magnetic Material, Volume 1: Fundamentals and Theory, pag. 95 (eds H. Kronmuller and S. Parkin) (Wiley, 2007).

14 P.W. Anderson, Science 235, 1196 (1987).

15 J.C. Xavier, Phys. Rev. B 68, 134422 (2003).

16 M.Z. Asadzadeh, F. Becca, and M. Fabrizio, Phys. Rev. B 87, 205144 (2013).

17 O. Bodensiek, R. Zitko, M. Vojta, M. Jarrell, and T. Pruschke, Phys. Rev. Lett. 110, 146406 (2013).

18 P. Coleman and A.H. Nevidomskyy, J. of Low Temp. Phys. 161, 182 (2010).

19 S. Nakatsuji, Y. Machida, Y. Maeno, T. Tayama, T.
Sakakibara, J. van Duijn, L. Balicas, J.N. Millican, R.T. Macaluso, and J.Y. Chan, Phys. Rev. Lett. 96, 087204 (2006).

20 M.S. Kim, M.C. Bennett, and M.C. Aronson, Phys. Rev. B 77144425 (2008).

21 Y. Motome, K. Nakamikawa, Y. Yamaji, and M. Udagawa, Phys. Rev. Lett. 105, 036403 (2010).

22 B.H. Bernhard, B. Coqblin, and C. Lacroix, Phys. Rev. B 83, 214427 (2011).

23 R. Peters, N. Kawakami, and T. Pruschke, J. Phys.: Conf. Ser. 320012057 (2011).

24 J.G. Rau and H.-Y. Kee, Phys. Rev. B 89, 075128 (2014).

${ }^{25}$ W. Wu and A.M.S. Tremblay, arXiv:1410.1496.

26 P. Coleman and N. Andrei, J. Phys.: Condens. Matter 1, 4057 (1989).

27 J.R. Iglesias, C. Lacroix, and B. Coqblin, Phys. Rev. B 56, 11820 (1997); B. Coqblin, C. Lacroix, M.A. Gusmao, and J.R. Iglesias, Phys. Rev. B 67, 064417 (2003).

28 M.D. Kim, C.K. Kim, and J. Hong, Phys. Rev. B 68, 174424 (2003).

29 J.C. Xavier and E. Dagotto, Phys. Rev. Lett. 100, 146403 (2008).

30 Y. Liu, H. Li, G.-M. Zhang, and L. Yu, Phys. Rev. B 86, 024526 (2012).

31 L. Isaev and I. Vekhter, Phys. Rev. Lett. 110, 026403 (2013).

32 N.K. Sato, N. Aso, K. Miyake, R. Shiina, P. Thalmeier, G. Varelogiannis, C. Geibel, F. Steglich, P. Fulde, and T. Komatsubara, Nature (London) 410, 340 (2001).

${ }^{33}$ H. Watanabe and M. Ogata, Phys. Rev. Lett. 99, 136401 (2007).

34 H. Aliaga, B. Normand, K. Hallberg, M. Avignon, and B. Alascio, Phys. Rev. B 64, 024422 (2001).

35 D.J. Garcia, K. Hallberg, B. Alascio, and M. Avignon, Phys. Rev. Lett. 93, 177204 (2004).

36 S. Sorella, Phys. Rev. B 71, 241103 (2005). 